\title{
Metodología GESINCA como instrumento de gestión empresarial en la industria camaronera venezolana
}

\author{
Trillo Holgado, María Amalia* \\ Espejo Mohedano, Roberto** \\ Hernández Escobar, Jean Antonio***
}

\begin{abstract}
Resumen
La metodología Gestión -Intangibles-Calidad (GESINCA) aplicada a la industria camaronera venezolana, se desarrolla con el objetivo de dotar a las empresas de un instrumento útil a la hora de canalizar sus decisiones estratégicas de acuerdo a las características de la economía actual. Basándose en los fundamentos teóricos relacionados con el capital intelectual y la calidad total, se establece un modelo de medición de intangibles que posibilita la visualización de los resultados obtenidos a partir de los movimientos estratégicos realizados por las empresas. La validación de la metodología a partir del modelo construido permite afirmar que el pilar básico sobre el que se asienta la gestión empresarial está formado por las capacidades organizativas y, que al intervenir sobre éstas se está potenciando el resto de capacidades (humanas, comerciales y sociales) y, por tanto, influyendo decisivamente en el futuro. Del mismo modo, se percibe una industria con un alto porcentaje de intangibles que merece la pena gestionar en aras a incrementar el valor de la misma.
\end{abstract}

Palabras clave: Calidad, capital intelectual, gestión empresarial, intangibles, modelos de gestión.

Recibido: 19-07-11. Aceptado: 06-07-12

* Doctora en Ciencias Económicas y Empresariales. Prof. Contratado Doctor del Área de Organización de Empresas de la Universidad de Córdoba, España. e-mail: maru.trillo@uco.es.

** Doctor en Informática y Licenciado en Matemáticas. Catedrático de Escuela Universitaria del Área de Estadística e Investigación Operativa de la Universidad de Córdoba, España. e-mail: roberto.espejo@uco.es

*** Asesor Laboral Corporativo en Standard Seafood de Venezuela. Magister Scientiarum en Gerencia de Empresas por la Universidad de Zulia, Venezuela. e-mail: jean_hernandez@hotmail.com 


\title{
GESINCA Methodology as a Managerial Instrument in the Venezuelan Shrimp Industry
}

\begin{abstract}
The methodology Management-Intangibles-Quality (GESINCA), applied to the Venezuelan shrimp industry, develops with the objective of giving the companies a useful instrument for channeling their strategic decisions according to current economic characteristics. Based on theoretical foundations related to intellectual capital and total quality, a model for measuring intangibles is established that makes it possible to visualize the results obtained from strategic movements carried out by the companies. Validation of the methodology using the constructed model made it possible to affirm that the basic pillar on which business management is established consists of organizational capacities, and when these are intervened, the other capacities (human, commercial and social) are empowered, thereby decisively influencing the future. In the same way, an industry is perceived with a high percentage of intangibles that merit management effort in order to increase its value.
\end{abstract}

Key words: Quality, intellectual capital, business management, intangibles, management models.

\section{Introducción}

En una economía mayoritariamente basada en el conocimiento en la que los clientes se tornan cada vez más exigentes, los activos intangibles y la calidad total constituyen piezas estratégicas fundamentales para la dirección de las organizaciones. Sin embargo, debido a la dificultad de valorar y gestionar los factores claves de éxito en este ámbito, las empresas aún tienen un largo camino por recorrer. Actualmente, se puede observar la práctica carencia de datos sobre intangibles en las memorias empresariales, por lo que se está trabajando a nivel internacional en el diseño de un informe estandarizado de capital intelectual que pueda ser presentado por las compañías a final de su ejercicio económico.

Con el objetivo principal de dotar a las organizaciones empresariales de un instrumento útil a la hora de canalizar sus decisiones estratégicas, conforme a las re- feridas nuevas tendencias del mercado, se desarrolla este trabajo, el cual aborda el diseño de un modelo de medición de intangibles basado en la calidad total desde la perspectiva del capital intelectual. Los pilares teóricos del mismo, por tanto, conciernen a la calidad y al capital intelectual. El modelo está formado por los siguientes elementos: capacidades humanas, organizativas, comerciales y sociales, así como por las tendencias del pasado y las expectativas futuras.

Posteriormente, se lleva a cabo una aplicación empírica del modelo diseñado, que toma como muestra a las empresas procesadoras de camarón que se encuentran activas en el Municipio de San Francisco del estado Zulia, Venezuela. Para ello, se aportan cinco escalas fiables y válidas que miden el carácter intangible de dichas entidades, así como un índice que permite establecer comparaciones entre las empresas que forman el sector, tal como se expone a continuación. 
Metodología GESINCA como instrumento de gestión empresarial en la industria... Trillo H., María Amalia; Espejo M., Roberto y Hernández E., Jean Antonio

La muestra de empresas considerada está integrada por doce organizaciones. Al tratarse de la población total, el error muestral del estudio es del $0 \%$ para el Estado de Venezuela e inferior al $1 \%$ si se considera todo el país. El trabajo de campo se ha llevado a cabo durante el período que abarca los meses de Noviembre de 2009 a Febrero de 2010.

Para medir los aspectos descritos en el modelo propuesto se han desarrollado y administrado a las empresas del sector estudiado las referidas cinco escalas Likert mediante un cuestionario único. La primera consta de diez preguntas relativas a las capacidades humanas $(\mathrm{HC})$; la segunda, con diez cuestiones también, trata de medir las capacidades organizativas (OC); una tercera escala, de nueve preguntas, hace referencia a las capacidades comerciales y sociales (CSC) y, finalmente, otras dos con seis preguntas cada una, enfocadas a la recogida de información relativa a tendencias surgidas en los últimos cinco años de actividad empresarial (PT), así como a las expectativas esperadas para el próximo año (FE). Por tanto, el modelo de cuestionario suministrado consta de cuarenta y una preguntas relativas a las distintas capacidades, así como a la recogida de información sobre el pasado y las predicciones para el futuro próximo. Cada una de las cinco escalas está formada por preguntas cerradas en las que sólo puede seleccionarse una opción, correspondiéndose con: 1 totalmente en desacuerdo, 2 en desacuerdo, 3 ligeramente en desacuerdo, 4 ligeramente de acuerdo, 5 de acuerdo y 6 totalmente de acuerdo.

El tratamiento estadístico de los datos se ha efectuado con el programa
SPSS para Windows V. 17.0., aplicándose distintas técnicas estadísticas de análisis, tal como se indica seguidamente.

Se ha verificado la fiabilidad de cada una de las escalas desarrolladas. Para ello, se ha evaluado la consistencia interna de cada elemento del modelo mediante el cálculo del coeficiente alfa de Cronbach.

Del mismo modo, se ha llevado a cabo un análisis factorial, para verificar la asignación de los ítems (preguntas del cuestionario) a las variables del modelo propuesto $\mathrm{y}$, por último, se ha hecho uso del coeficiente de correlación lineal de Pearson para analizar las relaciones entre los elementos del modelo propuesto.

Además, se han construido lo que se ha convenido en llamar índices GESINCA, que suponen la adaptación al modelo GESINCA de la metodología para el cálculo de índices definida por Trillo y Espejo (2008), posibilitando, así, la realización de comparativas entre las empresas objeto de estudio.

En definitiva, esta metodología permite: El control de la eficiencia de los procesos de gestión del conocimiento; el que las empresas o consultoras dispongan de la base de diseño de un software, que permita tener actualizada la información estratégica necesaria para la toma de decisiones de gestión; el cálculo de un índice a partir de información cualitativa y; el establecimiento de comparaciones entre empresas que se ubiquen en un mismo sector.

Por último, conviene resaltar que el interés de la metodología expuesta radica en su potencialidad para aplicarse a cualquier otro tipo de organizaciones. 


\section{Fundamentos teóricos para el diseño del modelo GESINCA}

Para alcanzar el éxito, las organizaciones, independientemente del sector donde se ubiquen, tamaño, estructura o madurez, necesitan establecer un sistema de gestión apropiado, tal como establece la European Foundation for Quality Management (EFQM, 2003), considerando que la creación de valor no sólo depende de los activos físicos, sino cada vez más de los intangibles.

En el contexto de los intangibles, el concepto de capital intelectual se torna protagonista. De hecho, muchos han sido los intentos por delimitarlo (Trillo y Sánchez, 2006) destacando su asimilación a activos intelectuales de la empresa, tal como señala la Internacional Management Accounting Study (IFAC, 1998), competencias básicas distintivas de carácter intangible (Bueno, 1998), intangibles, conocimientos o capital intelectual (Lev, 2001) combinación de recursos humanos, organizativos y relacionales (Meritum, 2002). Surgen, por tanto, diversos modelos de medición y gestión del capital intelectual en el ámbito internacional, como describe el Centro de Investigaciones sobre la Sociedad del Conocimiento (CIC, 2003), a través de los que se pueden valorar los intangibles de la empresa, que no se recogen desde el punto de vista contable y que, sin embargo, constituyen una fuente clave de creación de valor. En este sentido, algunas gran- des empresas como Microsoft, American Airlines y la empresa de Seguros Skandia (Edvinsson y Malone, 1997), entre otras, utilizan modelos propios.

Por otro lado, la consideración de la calidad en el ámbito empresarial ha tenido varias etapas que abarcan desde la mera inspección de productos a la gestión de la calidad total, pasado por el control, la planificación y la administración de la misma (Romero y Miranda, 2007).

Se parte de dos modelos que tienen una gran aplicación práctica en el mundo empresarial actual, además de abarcar, a nuestro entender, el abanico más amplio de los aspectos claves de la gestión de la empresa. Estos son: Intellectus $^{1}$, relativo al capital intelectual, y EFQM, relacionado con la calidad total que, por otra parte, tienen diversos puntos de conexión entre ellos, así como otros aspectos que resultan complementarios (Cuadro 1).

Se parte de la importancia de tener en cuenta el hecho de que las empresas puedan validar y gestionar la capacidad de su organización para alcanzar las metas propuestas en busca de la excelencia, obteniendo la información suficiente para, entre otras cosas, desarrollar nuevos proyectos, realizar innovación de productos y marcas, clarificar las alternativas de inversión en investigación y capacitación técnica, promocionar la cultura organizacional, sensibilizar respecto a la ética empresarial o definir actividades de negociación en el mercado.

1 El modelo Intellectus está actualmente en revisión al objeto de incorporar un capital más relacionado con la innovación. 
Metodología GESINCA como instrumento de gestión empresarial en la industria...

Trillo H., María Amalia; Espejo M., Roberto y Hernández E., Jean Antonio

\section{Cuadro 1 \\ Semejanzas y diferencias entre Intellectus y EFQM}

\begin{tabular}{|c|c|c|}
\hline & \multicolumn{2}{|c|}{ Modelo Intellectus y Modelo EFQM } \\
\hline \multirow{4}{*}{ 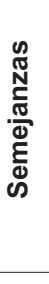 } & \multirow{4}{*}{\multicolumn{2}{|c|}{$\begin{array}{l}\text { - Son modelos integrales, abiertos y flexibles que pueden utilizarse como instrumento de } \\
\text { autoevaluación. } \\
\text { - Resultan útiles para la toma de decisiones estratégicas. } \\
\text { - Tienen en cuenta factores humanos, organizativos y relacionales. } \\
\text { - Ambos dan importancia clave a las relaciones tanto en el ámbito interno como externo } \\
\text { de la empresa. }\end{array}$}} \\
\hline & & \\
\hline & & \\
\hline & & \\
\hline \multirow{6}{*}{ 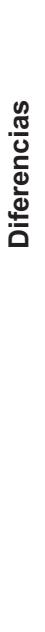 } & Modelo Intellectus & Modelo EFQM \\
\hline & $\begin{array}{l}\text { - Se centra en el desarrollo y gestión de ac- } \\
\text { tivos intelectuales. }\end{array}$ & $\begin{array}{l}\text { - Se centra en la implantación de los princi- } \\
\text { pios de gestión de la calidad total. }\end{array}$ \\
\hline & $\begin{array}{l}\text { - Su objetivo concierne al aprovechamiento } \\
\text { del capital intelectual de la empresa. }\end{array}$ & $\begin{array}{l}\text { - Su objetivo concierne al despliegue, resul- } \\
\text { tados, evaluación, revisión y perfecciona- } \\
\text { miento de lo que denomina conceptos } \\
\text { fundamentales de la excelencia. }\end{array}$ \\
\hline & $\begin{array}{l}\text { Estructura formada por los capitales: hu- } \\
\text { mano, organizativo, tecnológico, de nego- } \\
\text { cio y social. }\end{array}$ & $\begin{array}{l}\text { - Estructura que distingue entre agentes } \\
\text { facilitadores y resultados }\end{array}$ \\
\hline & $\begin{array}{l}\text { - Evalúa la estructura organizativa en el } \\
\text { momento actual. }\end{array}$ & $\begin{array}{l}\text { - La evaluación se basa en la detección de } \\
\text { puntos fuertes y puntos débiles. }\end{array}$ \\
\hline & $\begin{array}{l}\text { - Intenta formalizar y sistematizar los proce- } \\
\text { sos de identificación, administración y } \\
\text { control del capital intelectual. }\end{array}$ & $\begin{array}{l}\text { - Tiene una perspectiva empresarial estra- } \\
\text { tégica y gerencial con algunas derivacio- } \\
\text { nes tácticas. }\end{array}$ \\
\hline
\end{tabular}

Fuente: Elaboración propia.

Teniendo en cuenta los supuestos comentados anteriormente, el objetivo principal del modelo que se desarrolla consiste en proporcionar a las organizaciones un instrumento para la autoevaluación y el apoyo a la estrategia. A través del mismo se ponen de manifiesto los factores claves del éxito empresarial y las relaciones entre ellos. Del mismo modo, se proporcionan un número de indicadores manejable y representativo de dichos factores, que adaptados al caso concreto, deben ser objeto de medición (por medio del cuestionario aplicado) y evaluación periódica a fin de que la puesta en marcha de la estrategia de la empresa se lleve a cabo de una manera más eficaz.

El modelo que se plantea supone, por tanto, un avance respecto a los ya establecidos, ya que se dota de las siguientes características:

- Sencillez y capacidad de autodiagnóstico, en función del número y la representatividad de los indicadores propuestos.

- Facilidad de utilización, tanto por grandes como por pequeñas y medianas empresas, debido a su capacidad de adaptación al escoger aquellos indica- 
dores que resulten más representativos del caso que se estudie.

- Dinamicidad, pues tiene en cuenta el pasado y el futuro.

- Facilidad para establecer ponderaciones y grados de importancia relativas a los factores claves de éxito, contribuyendo así a una mayor exactitud a la hora de desarrollar y desplegar acciones estratégicas. Esto es posible a través del cálculo de un índice, tal como se explica más adelante, que permite hacer comparativas entre empresas.

- Posibilidad de visualizar la relación que se establece entre los factores de éxito para, de este modo, facilitar el despliegue de acciones encaminadas a su potenciación.

\section{EI modelo GESINCA (gestió-intangibles-calidad)}

Atendiendo a los principios básicos de gestión de intangibles y calidad total, así como a los modelos que han servido de referencia principal, se presenta el modelo GESINCA (gestión-intangibles-calidad) en el Diagrama 1.

Se observa que los elementos fundamentales de la gestión empresarial versan sobre el capital humano, organizativo y relacional, al modo en el que se considera por los modelos tradicionales de gestión de intangibles y que se desarrolla, de manera más detallada, en Intellectus. En este caso, se alude al concepto más amplio de capacidad y no al de capital, ya que con ello se quiere reflejar las competencias empresariales fruto del conocimiento y la experiencia con una perspectiva evolutiva.

GESINCA presenta el concepto de capacidades organizativas como el elemento más importante en cuanto que, dependiendo de una adecuada disposición de los recursos y un establecimiento procedimental, las capacidades humanas, así como las sociales y comer-

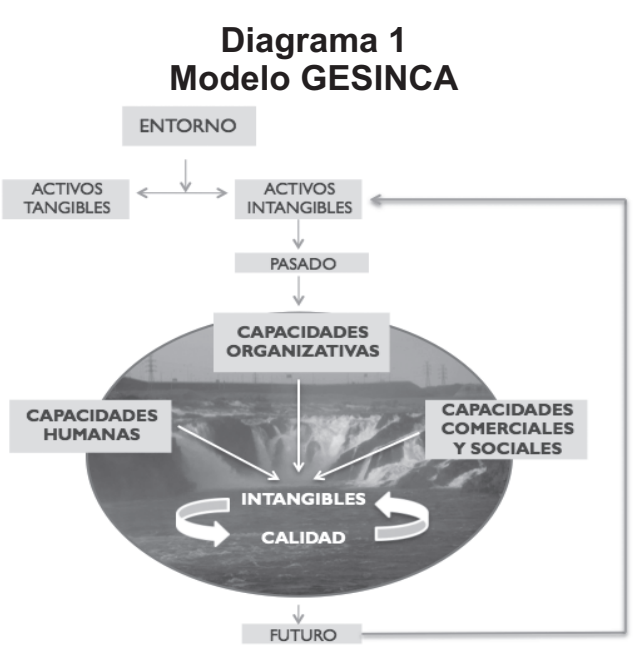

Fuente: Elaboración propia. 
Metodología GESINCA como instrumento de gestión empresarial en la industria... Trillo H., María Amalia; Espejo M., Roberto y Hernández E., Jean Antonio

ciales tienden a un desarrollo más ágil. Por otro lado, y tal como propone EFQM $(2003,2010)$, se dota a GESINCA de una dinamicidad que permite analizar la influencia de las estructuras pasadas en el futuro próximo y el proceso de feed-back al que se somete este ciclo.

GESINCA puede explicarse a través del detalle de sus principales componentes, que se desarrollan atendiendo básicamente a las teorías subyacentes en Intellectus y EFQM que se han tomado como referencia. Se describen a continuación:

Capacidades humanas: incluye los activos de conocimientos (tácitos o explícitos) y habilidades personales puestas al servicio de la organización en la que se trabaja.

La importancia de las capacidades humanas es corroborada a través de EFQM (2003) con afirmaciones como las que siguen: "excelencia es maximizar la contribución de los empleados a través de su desarrollo e implicación", "las organizaciones excelentes identifican y comprenden las competencias necesarias para implantar sus políticas, estrategias, objetivos y planes, tanto en la actualidad como en el futuro", "preparan a las personas para superar y adaptarse a cualquier cambio".

En la revisión del propio modelo EFQM (EFQM, 2010) se sigue realzando el papel de las personas, siendo uno de sus conceptos fundamentales "alcanzar el éxito mediante las personas", lo que se desarrolla "comprendiendo las habilidades y competencias necesarias para alcanzar la misión, visión y objetivos estratégicos", "alineando los objetivos individuales y de equipo" y "animando a las personas a ser creadoras y embajadoras del éxito continuado de la organización", entre otros.

En ambas versiones del modelo EFQM, se toman como agentes facilitadores de los resultados de la empresa tanto a las personas como al liderazgo.

Por otra parte, el capital humano se presenta como uno de los principales protagonistas en la mayoría de los modelos referidos a intangibles. Concretamente, en el modelo Intellectus ( $\mathrm{ClC}$, 2003) se define como "el valor de los conocimientos y del talento que poseen las personas que componen la organización".

Las variables e indicadores propuestos en el Cuadro 2, plasman la gran mayoría de aquellos que resultan esenciales, tanto en EFQM como en Intellectus. Respecto al primero, se recoge la motivación, satisfacción, logros y servicios prestados por la empresa a los empleados. En el modelo Intellectus, las variables más relacionadas con GESINCA son: sentimiento de pertenencia, satisfacción, flexibilidad y adaptabilidad, creatividad, aprendizaje, comunicación y liderazgo.

Capacidades organizativas: se refieren a las normas, procesos, estándares de calidad, tecnologías disponibles, patentes, marcas, métodos y procedimientos de trabajo, modelos, manuales, sistemas de dirección y gestión básicamente.

Respecto a las capacidades organizativas, EFQM (2003:8-9) afirma que la "excelencia es gestionar la organización mediante un conjunto de sistemas, procesos y datos interdependientes y relacionados", "excelencia es desafiar el status quo y hacer realidad el cambio aprovechando el aprendizaje para crear innovación y oportunidades de mejora". La idea se refuerza en EFQM (2010:17) al 


\begin{tabular}{|c|c|c|}
\hline \multicolumn{3}{|c|}{$\begin{array}{c}\text { Cuadro } 2 \\
\text { Componentes del modelo GESINCA }\end{array}$} \\
\hline Elementos & Variables & Indicadores \\
\hline \multirow[t]{4}{*}{$\begin{array}{l}\text { Capacidades } \\
\text { Humanas }\end{array}$} & $\begin{array}{l}\text { Ejemplaridad del } \\
\text { liderazgo (directivos) }\end{array}$ & $\begin{array}{l}\text { - Incentivos a la profesionalización. } \\
\text { - Comunicación directivos-empleados. } \\
\text { - Confianza directivos-empleados. }\end{array}$ \\
\hline & & $\begin{array}{l}\text { - Equilibrio objetivos individuales-empresa. } \\
\text { - Ambiente de trabajo. }\end{array}$ \\
\hline & $\begin{array}{l}\text { Capacitación estratégica } \\
\text { de los recursos } \\
\text { humanos }\end{array}$ & $\begin{array}{l}\text { - Definición perfil de competencias. } \\
\text { - Formación adaptada. } \\
\text { - Decisiones estratégicas. }\end{array}$ \\
\hline & $\begin{array}{l}\text { Gestión de la integración } \\
\text { de los trabajadores }\end{array}$ & $\begin{array}{l}\text { - Atención a las sugerencias/reclamaciones. } \\
\text { - Programas de integración. }\end{array}$ \\
\hline \multirow[t]{2}{*}{$\begin{array}{l}\text { Capacidades } \\
\text { Organizativas }\end{array}$} & $\begin{array}{l}\text { Gestión de } \\
\text { procesos }\end{array}$ & $\begin{array}{l}\text { - Definición de procesos claves. } \\
\text { - Integración estratégica de procesos. } \\
\text { - Eficiencia de los procesos. } \\
\text { - Establecimiento de mejores prácticas. } \\
\text { - Inversiones en equipos y métodos. } \\
\text { - Automatización de procesos. }\end{array}$ \\
\hline & $\begin{array}{l}\text { Gestión de la } \\
\text { innovación }\end{array}$ & $\begin{array}{l}\text { - Existencia de manuales de procedimiento. } \\
\text { - Software para la gestión del conocimiento. } \\
\text { - Incentivos a la innovación. } \\
\text { - Trabajo en equipo. }\end{array}$ \\
\hline \multirow[t]{3}{*}{$\begin{array}{l}\text { Capacidades } \\
\text { Comerciales } \\
\text { y Sociales }\end{array}$} & $\begin{array}{l}\text { Posicionamiento } \\
\text { comercial y social }\end{array}$ & $\begin{array}{l}\text { - Redefinición del posicionamiento de mercado. } \\
\text { - Programas de actuación frente a la comunidad y al } \\
\text { gobierno. }\end{array}$ \\
\hline & $\begin{array}{l}\text { Gestión de compras } \\
\text { y ventas }\end{array}$ & $\begin{array}{l}\text { - Gestión de las reclamaciones de clientes. } \\
\text { - Capacidad de respuesta ante los pedidos. } \\
\text { - Alianzas con proveedores. } \\
\text { - Acuerdos para compra de tecnología o mejora de } \\
\text { procesos. }\end{array}$ \\
\hline & $\begin{array}{l}\text { Atención al cliente } \\
\text { interno y externo }\end{array}$ & $\begin{array}{l}\text { - Programas de actuación en beneficio de clientes y } \\
\text { empleados. }\end{array}$ \\
\hline \multirow[t]{2}{*}{$\begin{array}{l}\text { Tendencias } \\
\text { del Pasado }\end{array}$} & $\begin{array}{l}\text { Planificación } \\
\text { estratégica }\end{array}$ & $\begin{array}{l}\text { - Procedimientos para identificar fortalezas y debili- } \\
\text { dades. } \\
\text { - Planificación de acciones de mejora por áreas. } \\
\text { - Adecuación de los objetivos planificados. }\end{array}$ \\
\hline & $\begin{array}{l}\text { Posicionamiento } \\
\text { sectorial }\end{array}$ & $\begin{array}{l}\text { - Tendencia positiva de los resultados clave. } \\
\text { - Comparación de resultados con el líder sectorial. } \\
\text { - Comparación de resultados con otros del sector. }\end{array}$ \\
\hline
\end{tabular}


Metodología GESINCA como instrumento de gestión empresarial en la industria...

Trillo H., María Amalia; Espejo M., Roberto y Hernández E., Jean Antonio

\section{Cuadro 2. Continuación}

\begin{tabular}{ccc}
\hline Elementos & Variables & Indicadores \\
\hline Expectativas & Priorización de & - Ponderación de fortalezas y debilidades. \\
Futuras & acciones estratégicas & - Priorización de acciones de mejora. \\
& - Resultados clave en evolución positiva. \\
& Objetivos y resultados & - Adecuación de objetivos. \\
& sectoriales & - Adecuación resultados en relación al líder en el \\
& sector. \\
& - Adecuación resultados en relación a otros del sector.
\end{tabular}

Fuente: Elaboración propia.

reiterar que "las organizaciones excelentes diseñan, gestionan y mejoran sus procesos, productos y servicios para generar cada vez mayor valor para sus clientes y otros grupos de interés". Esto se relaciona con el agente facilitador de los resultados empresariales referido a procesos.

Las capacidades organizativas se incluyen en los modelos tradicionales de capital intelectual en el denominado capital estructural, que en Intellectus se desglosa en capital organizativo y capital tecnológico.

En el caso del modelo GESINCA, se recoge la filosofía propuesta en EFQM (2003) en la que se habla de valorar la "introducción de las mejoras necesarias en los procesos mediante la innovación", así como de los diseños y gestiones de los mismos más adecuados para la generación de valor (EFQM, 2010). Del mismo modo, se incluyen la mayoría de las variables claves de Intellectus (CIC, 2003) tales como: pautas organizativas, creación y desarrollo de conocimiento, procesos dirigidos a grupos de interés, gastos en $1+\mathrm{D}+\mathrm{i}$, dotación de tecnología de producción, de información y de comunicaciones e innovación de procesos o de gestión.
Capacidades comerciales y sociales: son todas aquellas relaciones de la organización con los agentes de su micro y macroentorno.

Relacionado con este elemento, EFQM (2003, 2010) computa como agentes facilitadores a la estrategia de la empresa, así como a las alianzas y a los recursos, que trascienden en resultados respecto a los clientes y a la sociedad.

En los modelos tradicionales relativos a intangibles se habla de capital relacional, que el modelo Intellectus divide en capital de negocio y capital social, para discernir entre las relaciones que tienen que ver con los agentes asociados al negocio básico y las que se mantienen con otros agentes del entorno.

GESINCA asume la esencia de los protocolos de relación y gestión del EFQM $(2003,2010)$ que se relacionan con clientes, proveedores y sociedad general, así como las principales variables de Intellectus asociadas a este bloque de capacidades, que se pueden ejemplificar en: base de clientes relevantes, lealtad de los clientes, satisfacción de los clientes, procesos de relación con clientes, red de distribución, formaliza- 
ción de la relación con proveedores y capacidad de respuesta de proveedores, entre otros (CIC, 2003).

Tendencias del pasado y expectativas futuras: estos dos elementos son los que aportan dinamicidad al modelo, observándose cómo se produce un proceso de retroalimentación entre las estrategias desplegadas en el pasado y las futuras. Se describe el desarrollo de una planificación estratégica que tiene muy en cuenta el posicionamiento sectorial y la jerarquización de las acciones a emprender, conceptos estos dos últimos que son de esencial consideración en el modelo de calidad total.

\section{Aplicación empírica del modelo GESINCA}

Con el fin de validar la metodología Gestión -Intangibles- Calidad (GESINCA) aplicada a la industria camaronera venezolana, para que ésta sea útil a la hora de canalizar las decisiones estratégicas de las empresas del sector, tal como propone este trabajo, en este apartado se lleva a cabo un estudio estadístico que aplica diferentes técnicas de análisis y se procede al cálculo de un índice que permite la comparativa entre las diferentes organizaciones. Esto va a permitir la reflexión realizada en el apartado posterior relativa a los principales los factores que determinan la gestión estratégica de las empresas objeto de estudio.

Se puede afirmar que las escalas establecidas son consistentes internamente (Tabla 1). Para ello, se ha calculado el coeficiente alfa de Cronbach, que permite confirmar que todos los ítems de
Tabla 1 Consistencia interna

\begin{tabular}{lc}
\hline \multicolumn{1}{c}{ Dimensión } & $\begin{array}{c}\text { Alfa de } \\
\text { Cronbach }\end{array}$ \\
\hline Capacidades humanas (HC) & 0,832 \\
Capacidades organizativas (OC) & 0,926 \\
$\begin{array}{l}\text { Capacidades comerciales y } \\
\text { sociales (CSC) }\end{array}$ & 0,853 \\
$\begin{array}{l}\text { Tendencias del pasado -5 } \\
\text { últimos años- (PT) }\end{array}$ & 0,780 \\
$\begin{array}{l}\text { Expectativas futuras -próximo } \\
\text { año- (FE) }\end{array}$ & 0,702 \\
\hline
\end{tabular}

Fuente: Elaboración propia.

una escala miden lo mismo, el mismo concepto (elementos del modelo). A este respecto, se ha teniendo en cuenta a Nunnally y Bernstein (1994), que consideran como aceptables los valores del alfa de Cronbach iguales o mayores a 0.70 como indicativos de una buena consistencia interna.

Para determinar la validez de cada una de las escalas se ha procedido a la realización de un análisis factorial confirmatorio por medio del método de componentes principales y rotación varimax. El fin de este análisis es comprobar la idoneidad de la clasificación de las variables consideradas (Morales et al., 2003). Las Tablas 2 y 3 muestran la adecuación del método mediante el test de esfericidad de Bartlett (significativo al ser Sig. <0,001) y la varianza total explicada para cada capacidad.

El porcentaje medio de la varianza explicada por las variables en cada elemento del modelo es del 84,15\% para las capacidades HC, OC y CSC, y del $79,32 \%$ para PT y FE (Tabla 3 ). Este hecho pone de manifiesto la adecuada cla- 
Metodología GESINCA como instrumento de gestión empresarial en la industria...

Trillo H., María Amalia; Espejo M., Roberto y Hernández E., Jean Antonio

Tabla 2

Prueba de esfericidad de Bartlett para la cada elemento

\begin{tabular}{cccccc}
\hline & HC & OC & CSC & PT & FE \\
\hline Chi-cuadrado aproximado & 77,313 & 98,199 & 96,527 & 51,735 & 57,101 \\
gl & 45 & 45 & 36 & 15 & 15 \\
Sig. & 0,002 & 0,000 & 0,000 & 0,000 & 0,000 \\
\hline
\end{tabular}

Fuente: Elaboración propia.

Tabla 3

Varianza total explicada para cada elemento

\begin{tabular}{cccccccc}
\hline \multirow{2}{*}{ Elemento } & Variable & \multicolumn{3}{c}{ Autovalores iniciales } & \multicolumn{2}{c}{$\begin{array}{c}\text { Suma de las saturaciones al } \\
\text { cuadrado de la rotación }\end{array}$} \\
\cline { 3 - 8 } & & Total & $\begin{array}{c}\text { \% de la } \\
\text { varianza }\end{array}$ & $\begin{array}{c}\% \\
\text { acumulado }\end{array}$ & Total & $\begin{array}{c}\text { \% de la } \\
\text { varianza }\end{array}$ & $\begin{array}{c}\% \\
\text { acumulado }\end{array}$ \\
\hline \multirow{2}{*}{ HC } & 1 & 4,598 & 45,976 & 45,976 & 3,355 & 33,549 & 33,549 \\
& 2 & 1,912 & 19,121 & 65,097 & 2,640 & 26,404 & 59,952 \\
& 3 & 1,162 & 11,615 & 76,712 & 1,676 & 16,760 & 76,712 \\
OC & 1 & 6,196 & 61,956 & 61,956 & 3,834 & 38,340 & 38,340 \\
& 2 & 1,305 & 13,054 & 75,010 & 2,909 & 29,086 & 67,425 \\
& 3 &, 976 & 9,759 & 84,769 & 1,734 & 17,344 & 84,769 \\
CSC & 1 & 4,672 & 51,907 & 51,907 & 3,284 & 36,485 & 36,485 \\
& 2 & 2,304 & 25,603 & 77,510 & 2,680 & 29,781 & 66,266 \\
& 3 & 1,213 & 13,472 & 90,982 & 2,224 & 24,716 & 90,982 \\
PT & 1 & 3,061 & 51,011 & 51,011 & 2,820 & 46,997 & 46,997 \\
& 2 & 1,917 & 31,958 & 82,969 & 2,158 & 35,972 & 82,969 \\
FE & 1 & 2,400 & 40,005 & 40,005 & 2,281 & 38,024 & 38,024 \\
& 2 & 2,140 & 35,662 & 75,667 & 2,259 & 37,643 & 75,667 \\
\hline
\end{tabular}

Fuente: Elaboración propia.

sificación de las variables consideradas para medir los elementos del modelo.

También se ha realizado un análisis de correlaciones bivariadas entre los totales de cada capacidad mediante el coeficiente de correlación lineal de Pearson. Los resultados obtenidos se describen en la Tabla 4, mostrando correlaciones claramente significativas entre algunas de ellas (Sig. <0,05). Destaca la correlación existente del $\mathrm{OC}$ con $\mathrm{HC}$,
CSC y PT y, a su vez, la que se da entre $\mathrm{PT}$ y FE. Respecto a la correlación entre OC y CSC, conviene señalar que aunque aparece como no significativa al nivel del $5 \%$, sin embargo, sí que lo es al $8,3 \%$. En este punto hay que comentar que, el hecho de considerar el $5 \%$ de significación es una regla estándar y que la elección del nivel de significación depende de la naturaleza e interpretación de cada estudio. En este caso, al disponer de pocas 
empresas, se considera como significativa la correlación existente entre OC y CSC (Tabla 4).

Las puntuaciones descriptivas básicas de los totales de cada capacidad se presentan en la Tabla 5 . El $47,72 \%$ de las empresas obtuvo una puntuación igual o superior a la media en cada una de las capacidades, mostrando, de esta forma, las fortalezas de las empresas analizadas.

Finalmente, se ha construido el índice GESINCA como adaptación de la metodología de construcción y clasificación de índices de cultura empresarial definida por Trillo y Espejo (2008) a las empresas analizadas, que se calcula promediando, para cada empresa, las respuestas a las distintas preguntas homogeneizadas a través del criterio del cociente, que divide dichas respuestas entre los valores medios obtenidos de todas las respuestas que engloba un elemento. El resultado se dividirá entre el número de preguntas efectuadas
Tabla 5

Descriptivos básicos de cada elemento

\begin{tabular}{|c|c|c|c|c|}
\hline & Mínimo & Máximo & Media & Desv. típ. \\
\hline $\mathrm{HC}$ & 27,00 & 56,00 & 37,083 & 9,317 \\
\hline OC & 25,00 & 59,00 & 42,667 & 11,097 \\
\hline CSC & 25,00 & 50,00 & 39,727 & 8,149 \\
\hline $\mathrm{PT}$ & 17,00 & 32,00 & 21,583 & 4,870 \\
\hline FE & 18,00 & 33,00 & 27,000 & 4,472 \\
\hline
\end{tabular}

Fuente: Elaboración propia.

para cada elemento a fin de posibilitar la comparación entre ellos.

En analogía con Trillo y Espejo (2008) se establece como criterio de clasificación de empresas o sectores el siguiente: si el índice es superior a 1 se considera empresa muy fuerte en la capacidad, si está comprendido entre 0,8 y 1 implica que es fuerte, una capacidad aceptable se da con un índice entre 0,5 y 0,8 y, por último, si el índice es inferior a 0,5 , se considera débil.

Tabla 4

Correlaciones bivariadas entre elementos

\begin{tabular}{llccccc}
\hline & & HC & OC & CSC & PT & FT \\
\hline \multirow{2}{*}{ HC } & C. Pearson & 1 & $0,710\left(^{* *}\right)$ & 0,312 & 0,393 & 0,233 \\
& Sig. (bilateral) &. & 0,010 & 0,350 & 0,206 & 0,465 \\
OC & C. Pearson & $0,71\left(^{* *}\right)$ & 1 & $0,544(\#)$ & $0,704\left(^{*}\right)$ & 0,533 \\
& Sig. (bilateral) & 0,010 &. & 0,083 & 0,011 & 0,074 \\
CSC & C. Pearson & 0,312 & 0,544 & 1 & $-0,053$ & 0,272 \\
& Sig. (bilateral) & 0,350 & 0,083 &. & 0,877 & 0,418 \\
PT & C. Pearson & 0,393 & $0,704\left(^{*}\right)$ & $-0,053$ & 1 & $0,726\left(^{* *}\right)$ \\
& Sig. (bilateral) & 0,206 & 0,011 & 0,877 &. & 0,007 \\
FT & C. Pearson & 0,233 & 0,533 & 0,272 & $0,726\left(^{* *}\right)$ & 1 \\
& Sig. (bilateral) & 0,465 & 0,074 & 0,418 & 0,007 &. \\
\hline
\end{tabular}

$\left.{ }^{* *}\right)$ La correlación es significativa al nivel 0,01 (bilateral).

$\left(^{*}\right)$ La correlación es significativa al nivel 0,05 (bilateral).

(\#) La correlación es significativa al nivel 0,08 (bilateral).

Fuente: Elaboración propia. 
Metodología GESINCA como instrumento de gestión empresarial en la industria... Trillo H., María Amalia; Espejo M., Roberto y Hernández E., Jean Antonio

Del modo explicado previamente se obtienen los resultados que se muestran en las Tablas 6,7 y 8 .

En las Tablas 6 y 7 pueden observarse puntuaciones del índice superior a 1 , lo que demuestra, una vez más, las fortalezas de las empresas analizadas respecto a sus intangibles.

Por otro lado, la reglamentación del gobierno venezolano, orienta la aplicación de sus políticas a la profundización de un sistema socialista, obligando legalmente a las compañías a dirigir un porcentaje de sus ganancias a inversión

\begin{tabular}{|c|c|c|c|c|}
\hline \multicolumn{5}{|c|}{$\begin{array}{c}\text { Tabla } 6 \\
\text { Índices GESINCA para cada } \\
\text { empresa }\end{array}$} \\
\hline Empresa & IHC & $\mathrm{IOC}$ & ICCS & $\begin{array}{c}\text { Índice } \\
\text { GESINCA }\end{array}$ \\
\hline A & 0,97 & 0,71 & 0,92 & 0,87 \\
\hline B & 1,11 & 1,65 & 1,04 & 1,27 \\
\hline C & 0,88 & 0,88 & 0,79 & 0,85 \\
\hline D & 1,60 & 1,68 & 1,02 & 1,43 \\
\hline$E$ & 1,45 & 1,57 & 1,52 & 1,51 \\
\hline $\mathrm{F}$ & 1,28 & 1,40 & 1,58 & 1,42 \\
\hline G & 0,82 & 1,00 & 1,26 & 1,03 \\
\hline $\mathrm{H}$ & 0,77 & 0,94 & 1,26 & 0,99 \\
\hline I & 1,08 & 1,08 & 1,30 & 1,15 \\
\hline$J$ & 1,00 & 1,08 & 1,23 & 1,10 \\
\hline K & 0,94 & 1,28 & 1,33 & 1,18 \\
\hline $\mathrm{L}$ & 0,77 & 1,31 & 1,58 & 1,22 \\
\hline
\end{tabular}

Fuente: Elaboración propia. social. Por este motivo, en la Tabla 8 aparece el índice GESINCA del sector en dos versiones, la que considera todas las capacidades sin ponderar, y la que pretende aliviar el efecto ejercido por la política venezolana sobre las capacidades comerciales y sociales, ponderando éstas a la mitad respecto al resto de capacidades. Hay que señalar también que existe cualquier otra posibilidad de ponderación en función de la estrategia de la empresa.

\section{Tabla 8}

\section{Índices GESINCA sectoriales}

\begin{tabular}{ccc}
\hline Índice & Sin Ponderar & Ponderado \\
\hline Media & 1,17 & 0,96 \\
Clasificación & Muy Fuerte & Fuerte \\
\hline
\end{tabular}

Fuente: Elaboración propia.

Finalmente, la Tabla 9 muestra las correlaciones bivariadas entre los índices construidos y las tendencias del pasado relativas a los últimos cinco años, así como entre estos y las expectativas futuras para el próximo año, obteniendo las mismas conclusiones que las aludidas en la Tabla 4.

Llegado a este punto se puede afirmar que se ha aplicado el modelo GESINCA de medición de intangibles basado en el concepto de calidad total y capital intelectual, el cual posibilita un avance respecto a los ya existentes en función de su sencillez, dinamicidad y posibilidad de

Tabla 7

Índices GESINCA sectoriales según elementos

\begin{tabular}{cccc}
\hline Capacidades & $\begin{array}{c}\text { Humanas } \\
(\text { IHC) }\end{array}$ & $\begin{array}{c}\text { Organizativas } \\
(\text { IOC) }\end{array}$ & $\begin{array}{c}\text { Comerciales y } \\
\text { Sociales (ICSC) }\end{array}$ \\
\hline Media & 1,06 & 1,22 & 1,24 \\
Clasificación & Muy Fuerte & Muy Fuerte & Muy Fuerte \\
\hline
\end{tabular}

Fuente: Elaboración propia. 
Tabla 9

Correlaciones bivariadas entre índices, PT y FE

\begin{tabular}{llccccc}
\hline & & IHC & IOC & ICSC & PT & FE \\
\hline IHC & C. Pearson & 1 & $0,710\left(^{* *}\right)$ & 0,061 & 0,393 & 0,233 \\
& Sig. (bilateral) &. & 0,010 & 0,851 & 0,206 & 0,465 \\
\multirow{2}{*}{ IOC } & C. Pearson & $0,710\left(^{* *}\right)$ & 1 & 0,341 & $0,704\left(^{*}\right)$ & 0,533 \\
& Sig. (bilateral) & 0,010 &. & 0,277 & 0,011 & 0,074 \\
\multirow{2}{*}{ ICSC } & C. Pearson & 0,061 & 0,341 & 1 & $-0,188$ & 0,177 \\
& Sig. (bilateral) & 0,851 & 0,277 &. & 0,559 & 0,582 \\
PT & C. Pearson & 0,393 & $0,704\left(^{*}\right)$ & $-0,188$ & 1 & $0,726\left(^{* *}\right)$ \\
& Sig. (bilateral) & 0,206 & 0,011 & 0,559 &. & 0,007 \\
FE & C. Pearson & 0,233 & 0,533 & 0,177 & $0,726\left(^{* *}\right)$ & 1 \\
& Sig. (bilateral) & 0,465 & 0,074 & 0,582 & 0,007 &. \\
\hline
\end{tabular}

${ }^{(*)}$ La correlación es significativa al nivel 0,01 (bilateral).

$\left(^{*}\right)$ La correlación es significativa al nivel 0,05 (bilateral).

Fuente: Elaboración propia.

visualización de los resultados obtenidos a partir de movimientos estratégicos relativos a los factores clave de éxito empresarial. Del mismo modo, se ha realizado una aplicación empírica en las empresas procesadoras de camarón que se encuentran activas en el Municipio de San Francisco del estado Zulia, Venezuela. Con esto se ha cubierto el objetivo principal de este trabajo consistente en dotar a las referidas empresas de un instrumento procedimental sencillo a la hora de canalizar la toma de decisiones estratégicas y que, a su vez, tiene la posibilidad de aplicación a otras empresas en diferentes sectores.

Los resultados muestran que las escalas desarrolladas en el presente estudio son un instrumento con suficiente validez y fiabilidad para medir el carácter intangible pretendido. El análisis factorial confirma la existencia de tres factores (variables del aspecto a medir) en cada una de las dimensiones establecidas (capacidades) y que se corresponden con las variables propuestas en los diseños de las escalas, demostrando la validez de las mismas. Por otra parte, el cuestionario global usado ha demostrado tener una buena consistencia interna en cada una de las escalas, al disponer de un alfa de Cronbach superior a 0,70 en cada una de ellas.

Del mismo modo, se han detectado correlaciones significativas entre $\mathrm{OC} y$ $\mathrm{HC}$, CSC y PT, así como entre PT y FE. De esto se puede extraer que las $O C$ presentan un especial protagonismo como factor de influencia en el resto de las capacidades de la empresa incidiendo decisivamente en la marcha de la misma. Como consecuencia de lo anterior, a mayor OC mayor HC y CSC y, dado que OC también se correlaciona con PT y este con FE, puede inferirse que al intervenir sobre las capacidades organizativas de la empresa, estamos potenciando el resto de sus capacidades $y$, por tanto, influyendo decisivamente en el futuro. 
Metodología GESINCA como instrumento de gestión empresarial en la industria... Trillo H., María Amalia; Espejo M., Roberto y Hernández E., Jean Antonio

Además, las correlaciones bivariadas de las Tablas 4 y 9 coinciden mostrando la validez del procedimiento respecto a las relaciones que se identifican a través del modelo.

En definitiva, las relaciones detectadas y expuestas anteriormente ponen de manifiesto la adecuación del modelo GESINCA propuesto como instrumento para la gestión estratégica empresarial.

\section{Conclusiones}

Las inversiones realizadas en los últimos diez años por las empresas venezolanas que se relacionan con las capacidades humanas y organizativas, se consideran cada vez más reducidas debido a la contracción económica que afronta el país. Asimismo, el aumento de la inversión en capacidades comerciales se explica atendiendo a las diversas estrategias que formulan los líderes de las empresas para mantenerlas activas y evitar pérdidas en los ejercicios económicos. En este mismo sentido, la inversión en las capacidades sociales se debe a la reglamentación del gobierno venezolano, que orienta la aplicación de sus políticas a la profundización de un sistema socialista, obligando legalmente a las compañías a dirigir un porcentaje de sus ganancias a inversión social.

En definitiva, se puede afirmar que dentro del sector de las empresas procesadoras de camarón, las capacidades comerciales y sociales son las más valoradas, siendo las capacidades humanas las que se presentan más débiles.

Puede observarse que la gran mayoría de las empresas presentan índices GESINCA superiores a 1 , de hecho el $75 \%$ de las empresas tienen un índice
GESINCA muy fuerte $(>1)$, y el resto, fuerte $(>0,8)$, lo que demuestra las fortalezas de las empresas analizadas respecto a sus activos intangibles.

Del mismo modo, se pueden hacer las siguientes reflexiones estratégicas derivadas de los resultados obtenidos: las empresas E, F y D son las más fuertes del sector, mientras que $\mathrm{A}, \mathrm{C}$ y $\mathrm{H}$ son las empresas más débiles; la empresa $D$ sólo tiene que reforzar las capacidades comerciales y sociales para ponerse a la cabeza del mercado; si B refuerza las capacidades comerciales y sociales estaría también en una de las mejores posiciones del mercado; lo mismo le sucedería a K y $L$ pero reforzando capacidades humanas y a I, reforzando capacidades organizativas.

Es importante saber que las posiciones empresariales que están por debajo de los niveles medios del sector se encuentran en clara desventaja respecto a las que más sobresalen, ya que incluso una posición alrededor de los niveles medios dista bastante de la de aquellas empresas que más sobresalen.

Pese a la importancia de las capacidades organizativas y al tratarse de empresas del mismo sector, las mayores disparidades se producen en las capacidades organizativas, después en las capacidades humanas $\mathrm{y}$, por último en las capacidades comerciales y sociales.

El índice GESINCA para el sector muestra la fortaleza del mismo, incluso excluyendo el efecto de las políticas alentadoras de las capacidades comerciales y sociales.

En síntesis, en un país donde impera la crisis $y$, por tanto, las preocupaciones de las empresas son meramente financieras, las organizaciones poseen 
un conjunto realmente sustancial de intangibles que merece la pena gestionar para incrementar el valor de la las mismas. El modelo desarrollado describe un procedimiento que permite poner de manifiesto aquellas capacidades empresariales que resultan ser más débiles cara al mercado, a fin de poderlas potenciar desde un punto de vista competitivo.

\section{Referencias Bibliográficas}

Bueno, Eduardo (1998). "El capital intangible como clave estratégica en la competencia actual". Boletín de Estudios Económicos, Vol. 53. No. 164. pp. 207-229.

Centro de Investigación sobre la Sociedad del Conocimiento (2003). "Modelo Intellectus: medición y gestión del capital intelectual". Documentos Intellectus. No. 5. Madrid. Universidad Autónoma de Madrid. 172 pp.

Edvinsson, Leif y Malone, Michael Shawn (1997). Intellectual Capital: realizing your company's true value by dinding its hidden brainpower. New York. Harper Colling Publisher. 229 pp.

European Foundation for Quality Management (EFQM) (2003). Modelo EFQM de excelencia, versión para grandes empresas y unidades de negocios operativos. Bruselas. EFQM Publications. $35 \mathrm{pp}$.

European Foundation for Quality Management (EFQM) (2010). Modelo EFQM de excelencia. Bruselas. European Foundation for Quality Management. EFQM Publications. 34 pp.
International Management Accounting Study (IFAC) (1998). "The measurement and management of intellectual capital: an introduction". International Management Accounting Study, pp. 1-53.

Lev, Baruch (2001). Intangibles. Management, measurement and reporting. Washington. $1^{\text {st }}$ ed. Brookings Institute. 212 pp.

Morales, Pedro, Urosa, Blanca y Blanco Ángeles (2003). Construcción de escalas de actitudes tipo Likert. Madrid. La Muralla. $175 \mathrm{pp}$.

Nunnally Jum y Bernstein Ira (1994). Psychometric theory. New York. $3^{a}$ ed., McGraw-Hill. 754 pp.

Romero, Arturo Luis y Miranda Sandor Luis (2007). "La calidad, su evolución histórica y algunos conceptos y términos asociados". Disponible en web: http://www.gestiopolis.com/ administracion-estrategia/la-calidadhistoria-conceptos-y-terminosasociados.htm.Gestiopolis. (consultado el 10/02/09).

Trillo, María Amalia y Espejo, Roberto (2008). "Propuesta metodológica para establecer un índice de cultura organizacional a través del capital intelectual". Estudios de economía aplicada. Vol. 26. No. 2. pp. 105-118.

Trillo, María Amalia y Sánchez, Sandra (2006). "Influencia de la cultura organizativa en el concepto de capital intelectual". Revista Intangible Capital. Vol. 2. No. 11. pp. 164-180. 